\title{
A Horizontal-Pendulum Type Thrust Stand for Evaluating Higher Frequency Variation by Applying Acceleration Measurement to Null-Balance Method
}

\author{
By Yusuke YamauChI, ${ }^{1)}$ Yasuyuki YanO, ${ }^{2)}$ and Akira KAKAMI ${ }^{1)}$ \\ ${ }^{1)}$ Department of Mechanical Design Systems Engineering, University of Miyazaki, Miyazaki, Japan \\ ${ }^{2)}$ Technical Center, University of Miyazaki, Miyazaki, Japan
}

(Received June 23rd, 2017)

\begin{abstract}
We propose a horizontal-pendulum type thrust stand in which two-point acceleration measurement is applied to the null-balance method. The conventional displacement method was been applied to thrust measurement; thrust is determined by pendulum displacement which is caused by thrust. The null-balance method is also applied to the thrust measurement; a controller adjusts actuator force on the basis of pendulum displacement so as to null the pendulum displacement, and thrust is determined from actuator-driving current or voltage. However, both measurement methods can evaluate thrust variation up to one-third of the natural frequency that is determined by the configuration of the controller and pendulum. Hence, we proposed to apply acceleration measurement to the null-balance method, and showed that thrust variation could be measured up to $80 \mathrm{~Hz}$. However, the proposed methods showed errors beyond $80 \mathrm{~Hz}$; the error was caused by the translational flexibility of the torsional hinges. Then, we propose a new method that uses two-point acceleration measurement and adjustment of the center of gravity position to evaluate thrust variation beyond $80 \mathrm{~Hz}$. The prototype evaluated thrust variation accurately beyond $200 \mathrm{~Hz}$, although at $20-160 \mathrm{~Hz}$, underestimating the amplitudes of reference thrust by $50 \%$.
\end{abstract}

Key Words: Thrust Stand, Center of Gravity, Solenoid Actuator

\section{Nomenclature}

$a_{1}:$ acceleration measured accelerometer 1

$a_{2}:$ acceleration measured accelerometer 2

$c \quad$ : damping coefficient of pendulum for rotational motion

$F_{0} \quad$ : ratio of reference thrust to solenoid actuator driving current

$I_{m} \quad$ : moment of inertia around rotating axis of torsional hinge

I : moment of inertia around the Center of gravity

$i \quad$ : solenoid actuator driving current

$k_{d}:$ difference constant

$k_{i} \quad:$ integral constant

$k_{p}:$ proportional constant

$k \quad$ : torsional spring constant of torsional hinge

$l_{a 1} \quad: \quad$ length between torsional hinge and accelerometer 1

$l_{a 2}:$ length between torsional hinge and accelerometer 2

$l_{C G} \quad: \quad$ length between torsional hinge and center of gravity

$l_{s} \quad$ : length between torsional hinge and solenoid actuator

$l_{t} \quad: \quad$ length between torsional hinge and thruster

$T_{n u l} \quad: \quad$ thrust determined from actuator-driving current using the null balance method

$T_{\text {pro }}:$ thrust determined with proposed method with one-point acceleration measurement

$T_{\text {pro2 }}:$ thrust determined with proposed method with two-point acceleration measurement

$T_{\text {ref }}:$ reference thrust

$T \quad:$ thrust

$x_{h}$ : displacement originating from the translational flexibility of torsional hinges

$\Delta \quad:$ amplitude

\section{Introduction}

The continuous development of space engineering allows spacecraft to make various contributions to our society: communication, weather forecast and broadcasting. Today, more complex missions have been attracted; for example, formation flight enables a high resolution real time observation on the earth. However, such complex missions require precise control to maintain the relative distance between spacecraft. Hence, propulsion devices need to yield higher performance and have sufficient responsibility to thruster firing command. ${ }^{1)}$ From the points of view, thrust variation must be accurately evaluated in higher frequency region to clarify transient thrust variation at ignition and fluctuation during firing.

Conventionally, the displacement and null-balance methods have been applied to thrust measurement. In the former, thrust is determined from the pendulum displacement which is caused by thrust. In the latter, a controller adjusts actuator force so as to null the pendulum displacement. This design allows to determine thrust from actuator driving current or voltage, and to readily adjust measurable thrust range by tuning control parameters. However, these methods also have 
limitations in upper measurable frequency because of the resonance, resonant frequency of which is determined by pendulum configuration and control parameters. Hence, we proposed to apply acceleration measurement to the null-balance method, and showed that thrust variation was successfully measured up $80 \mathrm{~Hz}$, which was larger than a resonant frequency of $30 \mathrm{~Hz}^{2}{ }^{2}$

The upper frequency limitation of the proposed method is due to torsional hinges, which consisted of three flat springs and two metal cylinders in order to produce a restoring torque without yielding friction or hysteresis. However, the torsional hinges also produced an unnecessary translational flexibility. The torsional hinges had flat springs, which connected the two stainless steel tubes and yielded torques along the cylinder axis. The structure exhibited the translational flexibility; the torsional hinges produced restoring forces along the direction perpendicular to the cylinder axis. Our previous study showed that a spring constant of translational flexibility was $7.7 \times 10^{5}$ $\mathrm{N} / \mathrm{m}^{2}{ }^{2}$ As a result, the pendulum induced not only rotational motion along the torsional hinges but also translational motion in the directions perpendicular to the cylinder axis. The translational motion deteriorated thrust measurement accuracy above $80 \mathrm{~Hz}^{2}{ }^{2}$ Hence, to extend measurable frequency range, we proposed to place center of gravity (C.G.) of the pendulum on the hinge, and measure pendulum acceleration at two points. This design eliminates the influence of the translational flexibility. To show that the proposed thrust stand evaluate thrust beyond $80 \mathrm{~Hz}$, a thrust stand was prototyped and tested.

\section{Principles of Proposed Methods}

\subsection{Previously proposed method (one-point acceleration measurement)}

Figure 1 shows the method with one-point acceleration measurement. This is identical to the method that the authors have proposed. ${ }^{2-5)}$ The Newton equation for the thrust stand is described as

$$
I_{m} \ddot{\theta}+c \dot{\theta}+k \theta=l_{t} T+l_{s} F_{0} i .
$$

Using the proportional-integral-differential (PID) control, actuator-driving current of the solenoid actuator is expressed as

$$
i=-\left\{k_{p} \theta+k_{d} \dot{\theta}+k_{i} \int \theta d t\right\} .
$$

Pendulum displacement is nulled by selecting appropriate $k_{p}, k_{d}$ and $k_{i}$ so as to achieve $c \dot{\theta}+k \theta \approx 0$. Then, Eq. (1) is converted into

$$
T=\frac{I_{m}}{l_{t}} \ddot{\theta}-\frac{l_{s} F_{0}}{l_{t}} i .
$$

Accordingly, thrust $T$ is evaluable on the basis of actuator driving current $i$ and angular acceleration $\ddot{\theta}$.

\subsection{Newly proposed method (two-point acceleration measurement)}

Our previous study showed that the translational flexibility of the torsional hinge deteriorated the frequency response.2) Then, the thrust stand is modeled in terms of the translational flexibility. The Newton equations for the thrust stand are

$$
I \ddot{\theta}+c \dot{\theta}+k \theta=T\left(l_{t}-l_{C G}\right)+F_{o} i\left(l_{s}-l_{C G}\right)+k_{h} x_{h} l_{C G}
$$

$$
m \ddot{x}_{h}=F_{0} i+T-k_{h} x_{h} .
$$

Placing C.G. position on the rotational axis nulls a gap between C.G. and rotational axis $l_{C G}$, and hence eliminates the influence of the translational motion on Eq. (4).

The method with one-point acceleration measurement (previously proposed method) can not accurately evaluate $\ddot{\theta}$ because the accelerometer evaluated the sum of $l_{a} \ddot{\theta}$ and translational acceleration $\ddot{x}_{h}$. Hence, we propose to use two-point acceleration measurement to the method, as shown in Fig. 2. Measured accelerations are expressed as

$$
\begin{aligned}
& a_{1}=\ddot{x_{h}}+l_{a 1} \ddot{\theta} \\
& a_{2}=\ddot{x_{h}}-l_{a 2} \ddot{\theta} .
\end{aligned}
$$

Eliminating $\ddot{x}_{h}$ from Eq. (6) determines genuine $\ddot{\theta}$. Hence, in the newly proposed method, rotation and translation are separated in terms of pendulum motion and acceleration measurement.

\section{Prototyped Thrust Stand}

\subsection{Horizontal-pendulum type thrust stand}

Figure 2 shows a schematic of the prototype thrust stand. Two piezoelectric accelerometers were fixed on the pendulum, and their outputs were amplified by 50. A resonant-type displacement sensor, which measured pendulum displacement, constituted inductance-capacitance resonant circuit with the opposing metal plate. The gap between the displacement sensor and the plate varied the resonant frequency; this design yielded a resolution of $10 \mathrm{~nm}$.

\subsection{Control systems}

To null the pendulum displacement induced by thrust, a PID-controller was implemented in a control PC. The control PC calculated $\theta, \dot{\theta}$, and $\int \theta d t$ from the displacement sensor output, and determined the actuator-driving current. To reduce the noise in $\dot{\theta}$ originating from differentiating $\theta, \dot{\theta}$ was filtered using a finite-impulse-response (FIR) filter. PID parameters $\left(k_{p}=3000, k_{d}=130, k_{i}=15000\right)$ were selected on the basis the preliminary tests to stabilize the set-point control. ${ }^{6}$

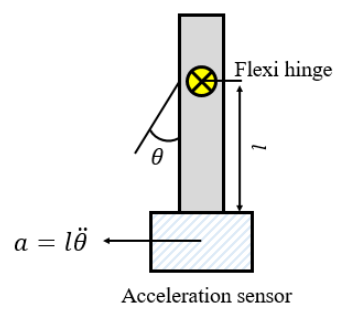

Fig. 1. Dynamic model of one-point acceleration measurement.

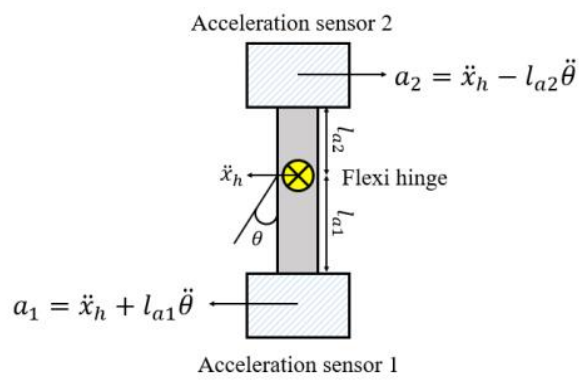

Fig. 2. Dynamic model of two-point acceleration measurement. 


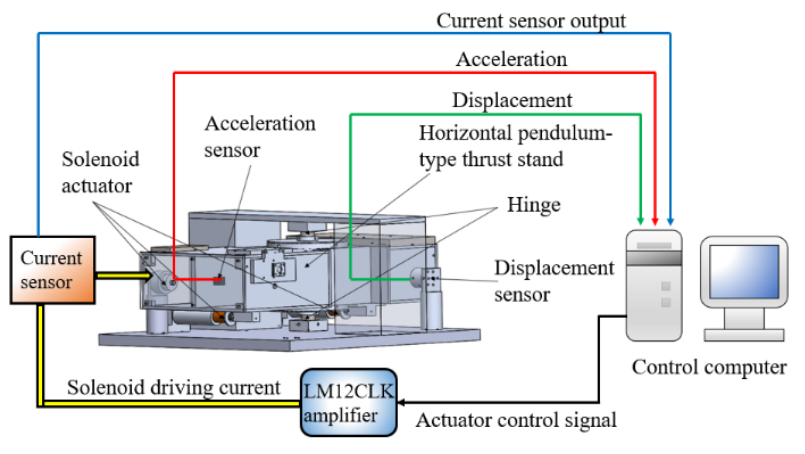

Fig. 3. Schematic of thrust stand.

\subsection{Calibration device}

Figure 3 shows a schematic of the designed calibration device. Three neodymium magnets, which were inserted into a hollow solenoid, were fixed on the cantilever of a load cell. Reference thrust i.e., electromagnetic force was exerted to the pendulum through the load cell; constant or biased sinusoidally-varying reference thrusts could be provided by adjusting actuator-driving current.

\subsection{Center of gravity position adjuster (C.G. adjuster)}

To accurately place the pendulum C.G position on the torsional hinge, we prototyped a C.G. position adjuster (Fig. 4). The C.G. position adjuster had two counterweights, which were moved along the guide by stepper-motors; appropriate counterweight position could eliminate position error of C.G. i.e., $l_{C G}$.

In order to evaluate $l_{C G}$, a C.G. position evaluator was prototyped (Fig. 5); the two vibrators, which were fixed on the base, exerted varying forces to the pendulum such that translational force was induced with zero net torque around the hinge by balancing force produced by the two vibrators. During force exertion, counterweight position was adjusted on the basis of $\ddot{\theta}$ and $\ddot{x}_{h}$, which were calculated using Eq. (6).

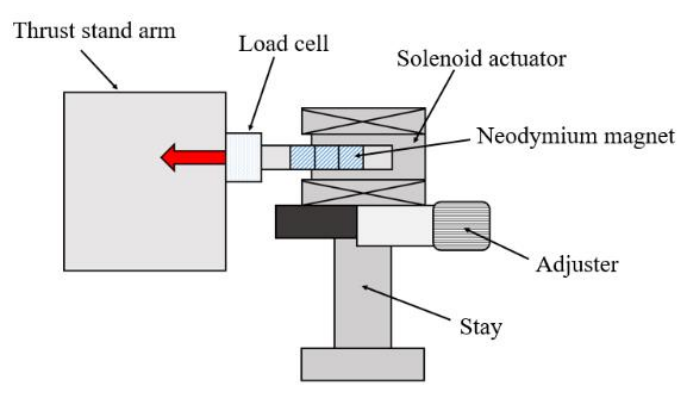

Fig. 4. Schematic of calibration device.
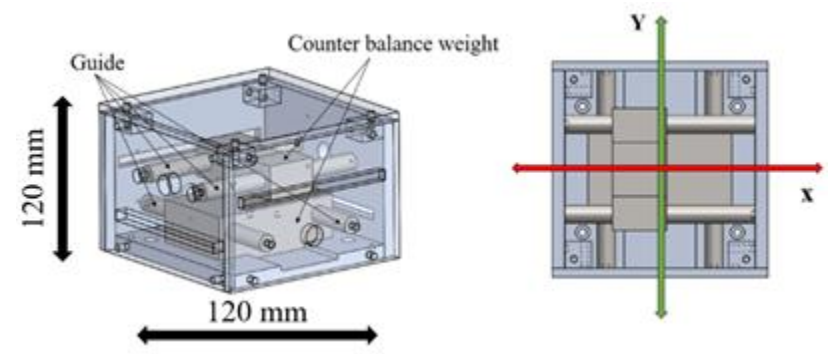

Fig. 5. Schematic of C.G. position adjuster.

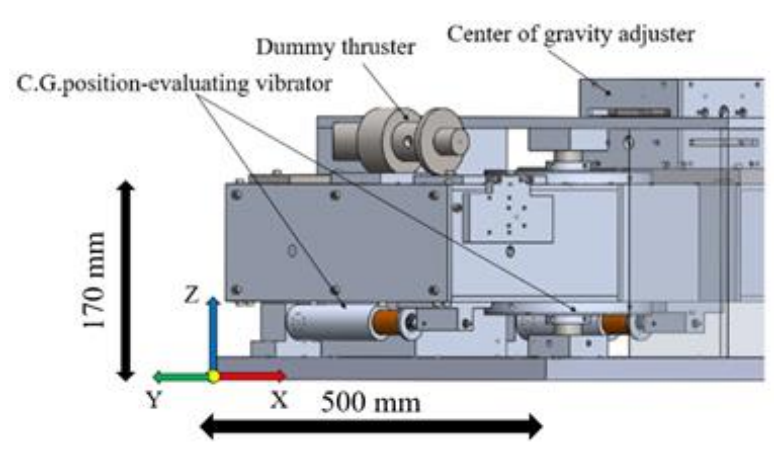

Fig. 6. Schematic of C.G. position evaluator.

\section{Experimental Produce}

\subsection{Static calibration}

In static calibration, the calibrating device exerted a constant reference thrust on the thrust-stand pendulum to evaluate the correlation between $i$ and constant thrust for the range of $0.1-1 \mathrm{~N}$.

\subsection{Frequency response evaluation}

Sinusoidally-varying thrust with offset was provided to thrust stand to evaluate frequency response. Offset and amplitude of thrust were $0.8 \mathrm{~N}$ and $0.3 \mathrm{~N}$; frequency ranged from $1 \mathrm{~Hz}$ to $350 \mathrm{~Hz}$. As in static calibration, reference thrust was measured by the load cell.

\section{Results and Discussion}

\subsection{C.G. position adjustment}

The C.G. position evaluator exerted forces at the two points on the pendulum such that net torque was zero to examine whether the C.G. position adjustment could eliminate the influence of $l_{C G}$ on translational motion. Figure 6 shows a time history of $\ddot{\theta}$ and $\ddot{x}_{h}$ for theoretical $l_{C G}=5 \mathrm{~cm}$; despite zero net torque around the hinge, rotational vibration was induced. By contrast, as shown in Fig. 7, wherein C.G. is theoretically located at the flexible hinge, $\ddot{\theta}$ is almost nulled. From these results, the effect of translational motion on the rotational motion could be eliminated by the adjustment of the C.G. position.

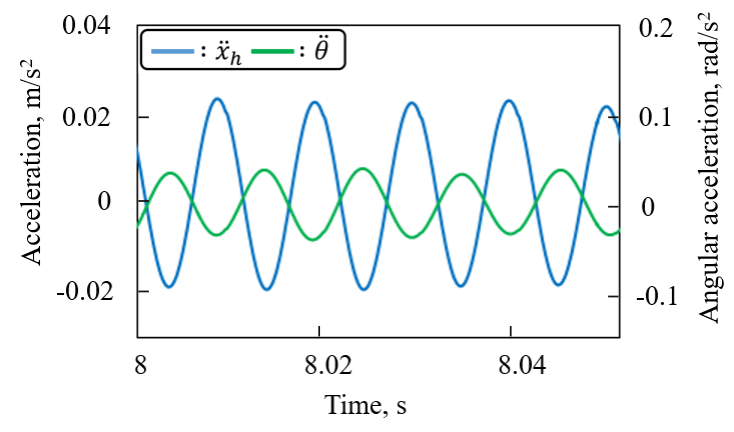

Fig. 7. Time history of $\ddot{x}_{h}$ and $\ddot{\theta}$ for theoretical $l_{C G}=5 \mathrm{~cm}$. 


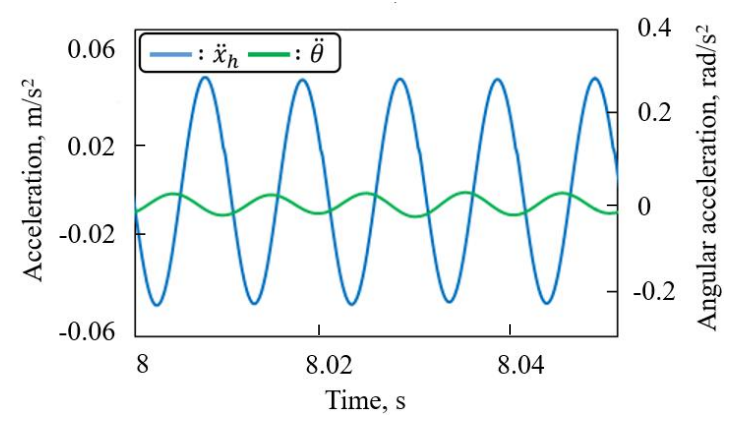

Fig. 8. Time history of $\ddot{x}_{h}$ and $\ddot{\theta}$ for theoretical $l_{C G}=0 \mathrm{~cm}$.

\subsection{Static calibration results}

Figure 8 shows the correlation between $i$ and $T_{\text {ref }}$ in the static calibration. Current-sensor output, which is linear to $i$, is linearly increased with $T_{r e f}$ with a coefficient of determination $R^{2}$ of 0.9986 . Hence, the prototype thrust stand can measure constant thrust $(0 \mathrm{~Hz})$.

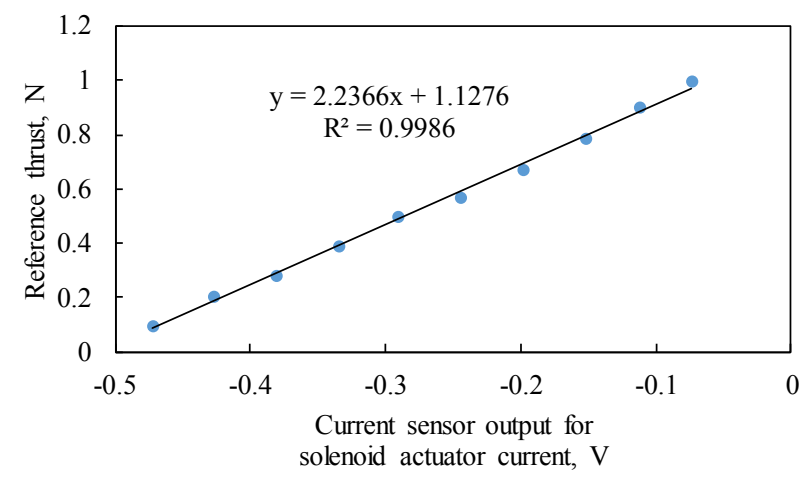

Fig. 9. Correlation between reference thrust and current sensor output voltage for actuator driving current.

\subsection{Dynamic response}

Figures 9-12 show time histories of $T_{\text {pro2 }}, T_{\text {pro }}, T_{\text {nul }}$ and $T_{\text {ref }}$. At $2 \mathrm{~Hz}, T_{\text {nul }}, T_{\text {pro }}$, and $T_{\text {pro } 2}$ are consistent with $T_{\text {ref }}$ without presenting any phase delay. However, at $40 \mathrm{~Hz}, T_{n u l}$ exhibited errors; $T_{\text {pro }}$ and $T_{\text {pro } 2}$ have smaller amplitude than $T_{\text {ref }}$ by $50 \%$ but have no phase delay (Fig. 10). In the range of $50-160 \mathrm{~Hz}$, the evaluated thrusts had errors as in $40 \mathrm{~Hz}$. At $220 \mathrm{~Hz}$ (Fig. 11), $T_{n u l}$ was maintained at the offset of $T_{r e f}$ and exhibited no amplitude, and $T_{\text {pro }}$ had a larger amplitude than that of $T_{\text {ref }}$ by a factor of 1.87 with phase delay. By contrast, $T_{\text {pro2 }}$ exhibited insignificant difference from reference thrust. At $350 \mathrm{~Hz}, T_{\text {null }}, T_{\text {pro } 1}$, and $T_{\text {pro } 2}$ present the same tendency as those at $220 \mathrm{~Hz}$. From these results, the proposed method with the two-point acceleration measurement could extend upper measurable frequency.

\subsection{Frequency response}

Figure 13 shows the frequency response of evaluated thrusts. Amplitude ratio expresses the amplitude ratio of the evaluated thrusts to reference thrust; the ideal value is 1 , wherein the evaluated amplitude is completely consistent with that of the reference; amplitude ratios of less and more than 1 represent underestimation and overestimation, respectively. The null-balance method accurately evaluated varying thrust up to $4 \mathrm{~Hz}$, but overestimated for more than one-third of the resonant frequency that is determined by the configurations of the PID controller and pendulum. At more than the resonant frequency of $8 \mathrm{~Hz}, \Delta T_{\text {nul }} / \Delta T_{\text {ref }}$ monotonically decrease; this means that the null-balance method is more insensitive to thrust variation as the frequency increases, and finally evaluate only the offset of $T_{r e f}$. Hence, $\Delta T_{n u l} / \Delta T_{\text {ref }}$ was lower than the minimum value of the longitudinal axis above $40 \mathrm{~Hz}$.

$T_{\text {pro }}$ and $T_{\text {pro } 2}$ were consistent with $T_{\text {ref }}$ up to $9 \mathrm{~Hz}$, however, the both proposed methods underestimated reference thrust by $50 \%$ for $20-160 \mathrm{~Hz}$, and overestimated at $180 \mathrm{~Hz}$. For more than $200 \mathrm{~Hz}$, whereas $T_{\text {pro1 }}$ exhibited overestimation with phase delay, $T_{\text {proz }}$ accurately followed $T_{r e f}$. From these results, the proposed method with two-point acceleration measurement and C.G. position adjustment successfully extended upper measurable frequency.

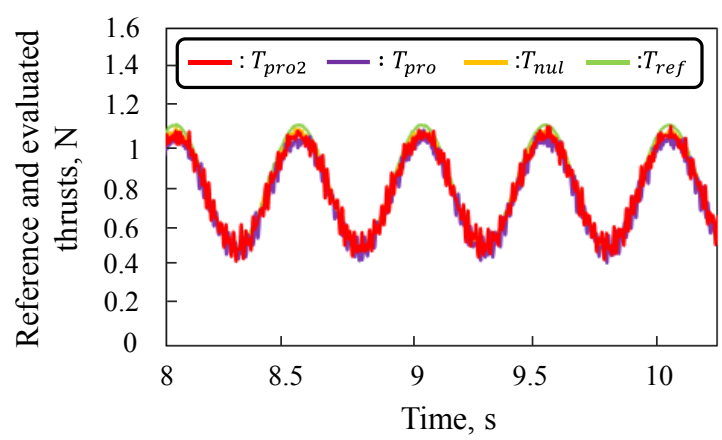

Fig. 10. Time history of thrusts $T_{\text {pro2 }}, T_{\text {pro }}, T_{\text {nul }}, T_{\text {ref }}$ for biased sinusoidally-varying reference thrust of $2 \mathrm{~Hz}$ in frequency.

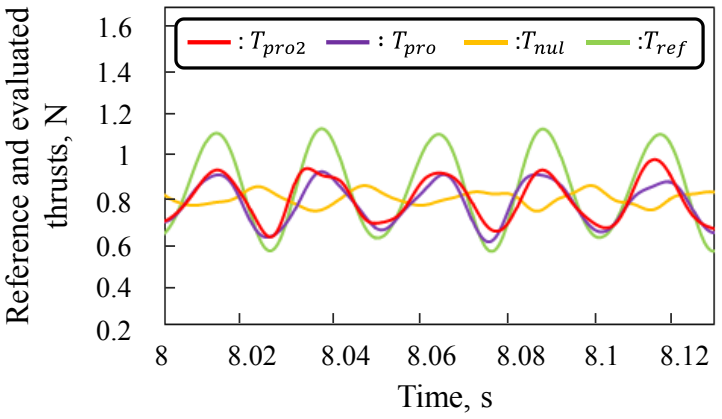

Fig. 11. Time history of thrusts $T_{\text {pro2 }}, T_{\text {pro }}, T_{\text {nul }}, T_{\text {ref }}$ for biased sinusoidally-varying reference thrust of $40 \mathrm{~Hz}$ in frequency.

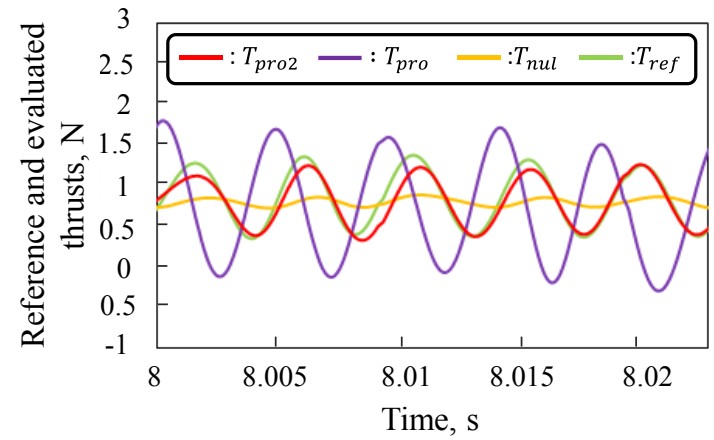

Fig. 12. Time history of thrusts $T_{\text {pro2 }}, T_{\text {pro }}, T_{\text {nul }}, T_{\text {ref }}$ for biased sinusoidally-varying reference thrust of $220 \mathrm{~Hz}$ in frequency. 


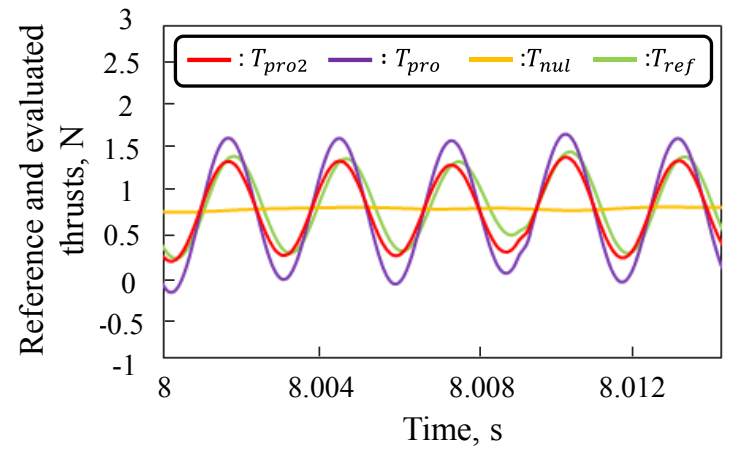

Fig. 13. Time history of thrusts $T_{\text {pro2 }}, T_{\text {pro }}, T_{\text {nul }}, T_{\text {ref }}$ for biased sinusoidally-varying reference thrust of $350 \mathrm{~Hz}$ in frequency.

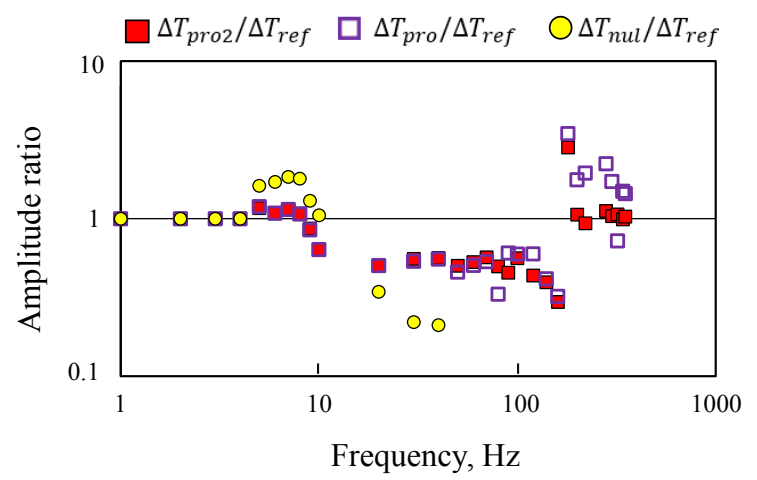

Fig. 14. Dependence of amplitude ratios $\Delta T_{\text {proz }} / \Delta T_{\text {ref }}, \Delta T_{\text {pro }} / \Delta T_{\text {ref }}$, $\Delta T_{n u l} / \Delta T_{r e f}$ on frequency.

\subsection{Discussion}

The proposed methods yield amplitude errors in the range of 20-180 Hz. As shown in Fig. 13, in the frequency range, the null-balance method was insensitive to varying thrust, and then thrust variation was evaluated using acceleration. Hence, a portion of thrust variation was possibly absorbed by some elements of the thrust stand.

Here, we focused on $c$ and $k$ of the thrust stand. As mentioned earlier, we assumed that $c \dot{\theta}+k \theta$ is ignorable owing to the set-point control. If set-point control tuning is inappropriate, $c \dot{\theta}+k \theta$ would negatively affect thrust measurement accuracy. Then, $c \dot{\theta}$ and $k \theta$ was evaluated. ${ }^{6}$

Figures 14 and 15 show time history of angular displacement and velocity at $20 \mathrm{~Hz}$ where the amplitudes of $\dot{\theta}$ and $\theta$ were the largest among the tests. From the result, the maximum values of $c \dot{\theta}$ and $k \theta$ are $0.00165 \mathrm{~N}$ and $0.013 \mathrm{~N}$ respectively. Hence, $c \dot{\theta}+k \theta$ had slight influence on $1 \mathrm{~N}$ class thrust measurement.

The deterioration is attributable to the translational flexibility of the torsional hinge and slight position errors of C.G. from the rotational axis. In the proposed method, the effect of translational motion is assumed to be eliminated by the position adjustment of C.G. of the pendulum, but Fig. 7 shows that rotational motion was affected by the translation forces. Hence, the misalignment of C.G. position would have been still present in the thrust stand. To examine the assumption, the natural frequency of the pendulum translational motion was evaluated by calculating translational spring constant of the torsional hinges.

To gain the translational spring constant of the torsional hinges, we analyzed a three dimensional model shown in Fig. 16 using Finite Element Method (FEM) software bundled with Solid Works. Figures 17 and 18 show the results of static analysis; the translational displacement is linearly increased with external force, and spring constant is $874.28 \times 10^{4} \mathrm{~N} / \mathrm{m}$. The natural frequency of the pendulum translational motion is determined to be $187.95 \mathrm{~Hz}$ from the spring constant and a pendulum mass of $12.54 \mathrm{~kg}$. The natural frequency is in the range where the accuracy was deteriorated in Fig. 13. Hence, the pendulum and the torsional hinge constituted an oscillator, which reduced amplitude ratio in the range of 20-160 Hz and increased the value in $160-180 \mathrm{~Hz}$. From the results, more precise adjustment of C.G. position would enhance the accuracy of the proposed method.

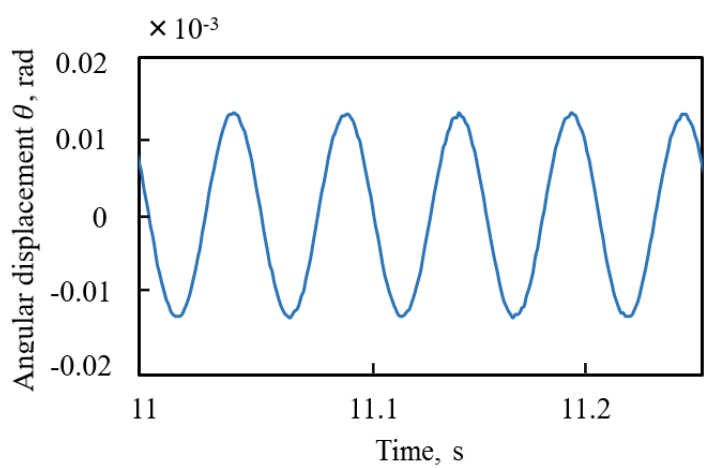

Fig. 15. Time history of angular displacement in $20 \mathrm{~Hz}$.

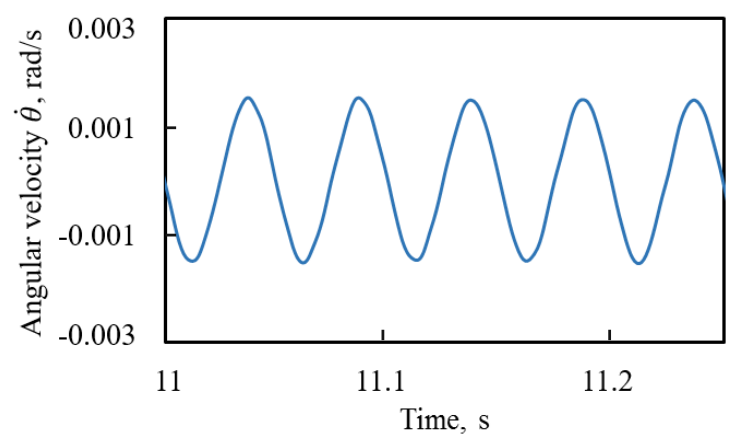

Fig. 16. Time history of angular velocity in $20 \mathrm{~Hz}$.

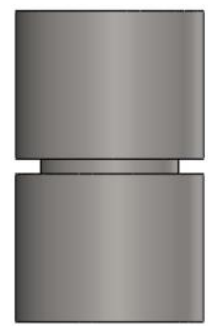

$\phi 25 \mathrm{~mm}$

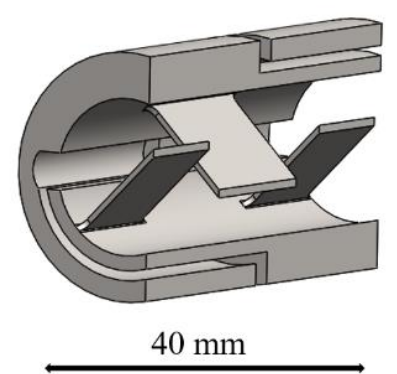

Fig. 17. Schematic of torsional hinge. 


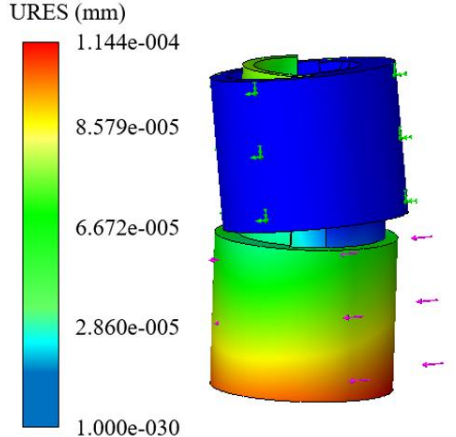

Fig. 18. Result of static analysis of torsional hinge.

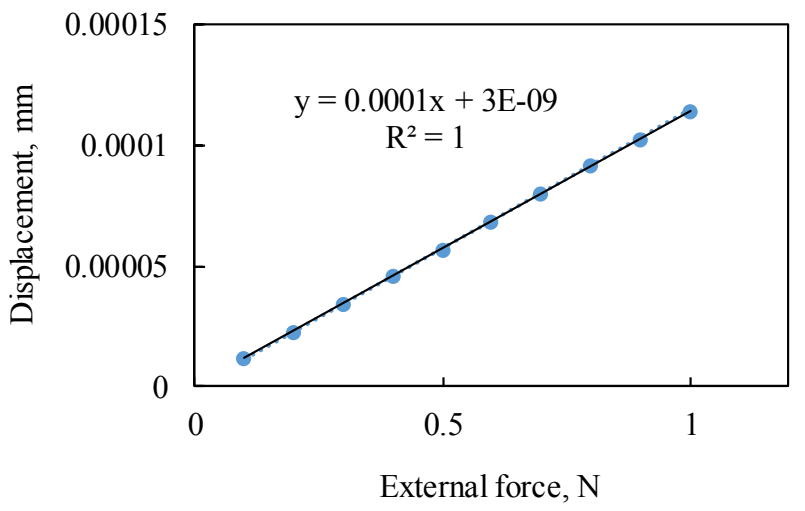

Fig. 19. Correlation between displacement and external force.

\section{Summary}

1. We proposed a thrust measurement method with the two-point acceleration measurement and C.G. position adjustment to accurately evaluate thrust beyond $80 \mathrm{~Hz}$.

2. The null-balance method accurately followed varying reference thrust up to $4 \mathrm{~Hz}$, but yield errors in amplitude and phase at more than one-third of the resonant frequency.

3. The proposed method with one-point acceleration measurement accurately evaluated varying thrust up to 9 $\mathrm{Hz}$, but, underestimated the thrust by $50 \%$ in the range of $20-160 \mathrm{~Hz}$, and overestimated it for more than 200 $\mathrm{Hz}$.

4. The proposed method with two-point acceleration measurement and C.G. position adjustment accurately evaluated varying thrust up to $9 \mathrm{~Hz}$, but, underestimated by $50 \%$ at the range of $20-160 \mathrm{~Hz}$. For $200-350 \mathrm{~Hz}$, thrust was correctly evaluated without error in phase and amplitude.

5. The unknown elements could act like a dynamic damper with a resonant frequency of $20-180 \mathrm{~Hz}$, where the proposed methods yield errors. This would deteriorate the accuracy in the frequency range of $20-180 \mathrm{~Hz}$.

\section{References}

1) George, P. S. and Oscar, B.: Rocket Propulsion Elements, John Wiley \& Sons, New York, 2010, pp. 14-22.

2) Kakami, A., Kashihara, K., Takeshida, S. and Yano, Y.: A New
Thrust Measurement Method for Evaluating Higher Frequency Variation by Applying Acceleration Measurement to Null-balance Method, Trans. JSASS Aerospace Tech. Japan, 14, ists30 (2015), pp. Pb_123-Pb_130.

3) Kakami, A., Hiyamizu, R., Masaki, S. and Tachibana, T.: A Preliminary Study on an Active-controlled Thrust Stand for Thrust Variation Measurement, Trans. JSASS Aerospace Technology Japan, ists25 (2006), pp. 212-217.

4) Kakami, A. and Tachibana, T.: Thrust Evaluation in Wide Frequency Range using Active Control and Disturbance Observer, J. Propulsion and Power, 29 (2013), pp. 1274-1281.

5) Kakami, A. and Tachibana, T.: Measurement of Thrust with Wide-Frequency Range Variation by Applying Active Control and Disturbance Observer, Aerospace Tech. Japan, 60 (2012), pp. 203-211 (in Japanese).

6) Bissel, C.C.: Control Engineering, Chapman \& Hall, New York, 1996, pp. 149-170. 